
\title{
GrNeCOLOCICACACANCER Updates and management algorithm for neuroendocrine tumors of the uterine cervix
}

${ }^{1}$ Department of Gynecologic Oncology and Reproductive Medicine, University of Texas MD Anderson Cancer Center, Houston, Texas, USA ${ }^{2}$ Department of Medical Oncology, Clinica Universidad Navarra, Madrid, Spain

Correspondence to Dr Gloria Salvo, Department of Gynecologic Oncology and Reproductive Medicine, University of Texas MD Anderson Cancer Center, Houston, TX 77030, USA; glorietasalvo@gmail.com; GSalvo@mdanderson.org

Received 29 March 2019 Revised 14 May 2019 Accepted 15 May 2019

\section{Check for updates}

C) IGCS and ESG0 2019. No commercial re-use. See rights and permissions. Published by BMJ.

\section{To cite: Salvo $G$}

Gonzalez Martin A

Gonzales NR, et al. Int J

Gynecol Cancer 2019;29:986995.

Gloria Salvo, ${ }^{\top 1}$ Antonio Gonzalez Martin, ${ }^{2}$ Naomi R Gonzales, ${ }^{1}$ Michael Frumovitz ${ }^{1}$

\begin{abstract}
Neuroendocrine carcinomas of the cervix account for less than $2 \%$ of all invasive cervical cancers and are classified as low-grade (carcinoid, atypical carcinoid tumor) or highgrade (known as small- and large-cell) neuroendocrine carcinomas. There are increasing data showing that cervical neuroendocrine carcinomas may be associated with the human papillomavirus (HPV), especially HPV18, and most will stain positive for p16. Immunohistochemistry markers such as synaptophysin and CD56 are the most sensitive markers. Although there are no commonly associated mutations, PIK3CA, KRAS, and TP53 are the most frequently found mutations in neuroendocrine tumors. Neuroendocrine cervical carcinomas are exceedingly aggressive tumors with a high tendency for nodal involvement and distant metastases. Age, lymph node metastases, smoking, pure small-cell histology, and tumor size are independent prognostic factors. Overall, the 5 -year survival rate is $36 \%$ and the median overall survival ranges between 22 and 25 months. Treatment options are often extrapolated from small-cell lung cancer and limited retrospective studies. The preferred treatment is a multimodal approach of surgery, chemoradiation, and systemic chemotherapy. The most common chemotherapy regimen used as initial therapy is a combination of cisplatin and etoposide. In the setting of recurrent disease, a combination of topotecan, paclitaxel, and bevacizumab has demonstrated favorable outcomes. Multicenter tumor registries, such as the Neuroendocrine Cervical Tumor Registry (NeCTuR), are an opportunity to evaluate patterns of disease treatment and oncologic outcomes.
\end{abstract}

\section{BACKGROUND}

Neuroendocrine tumors are a rare entity of the female genital tract, with cervix being the most common primary site. ${ }^{1}$ First described by Albores-Saavedra in 1972 , these tumors account for $1.4 \%$ of all invasive cervical cancers and nearly 200 new cases are diagnosed in the United States each year. ${ }^{23}$ The 2014 World Health Organization (WHO) Classification ${ }^{2}$ categorizes cervical neuroendocrine tumors as low-grade (previously referred to as carcinoid tumor and atypical carcinoid tumor) or high-grade neuroendocrine carcinomas (previously referred to as small-cell carcinoma or large-cell neuroendocrine carcinoma) (Table 1). In the cervix, small-cell neuroendocrine tumor is the most common (80\%) followed by large-cell neuroendocrine carcinoma (12\%), and other histologic types such as undifferentiated neuroendocrine tumors (8\%).

Unlike squamous and adenocarcinoma subtypes, which spread primarily by local extension, high-grade neuroendocrine tumors have a high rate of lymphatic and hematogenous metastasis even when disease is clinically limited to the cervix. These tumors frequently have lymph vascular space involvement, strong association with HPV18, and an increased risk of nodal metastases at diagnosis, with $40 \%$ of clinical stage IB1 tumors diagnosed with positive pelvic nodes, in comparison to $10 \%-15 \%$ node positivity seen in clinical stage IB1 squamous cell carcinoma. ${ }^{3}{ }^{4}$ Patients with small-cell carcinoma are more likely to be diagnosed at late stage. ${ }^{5}{ }^{6}$ Extra-pelvic disease at the time of diagnosis is also common, especially in the lungs and liver, and is often associated with high risk of relapse. ${ }^{78}$ The median overall survival for patients with small-cell neuroendocrine tumors is less than 2 years. $^{569-11}$

There is a total of 3,538 cases of neuroendocrine cervical cancers reported in 147 studies in the literature to date and only nine studies included more than 50 patients. $^{3}$ The lack of prospective studies limits our ability to define concrete chemotherapy regimens, and the primary treatment is a multimodal approach including surgery, radiation, and chemotherapy. ${ }^{12} 13$

At MD Anderson Cancer Center we have a registry of neuroendocrine cervical carcinomas with patients treated not only at our institution but also elsewhere. All neuroendocrine cervical carcinomas are treated by the same small group of physicians that also serve as consultants for doctors from around the world. In this review we aim to summarize the latest and most relevant information regarding this disease and also provide our algorithms for diagnosis and treatment. In addition, we will discuss key points to help physicians around the world provide better treatment for this uncommon disease.

\section{ETIOLOGY}

The association between squamous cell carcinoma, adenocarcinoma, and adenosquamous carcinoma of the cervix with human papillomavirus (HPV) has been well established and documented. ${ }^{14}{ }^{15}$ For cervical neuroendocrine carcinomas, the link with HPV has not 
Table 1 World Health Organization (WHO) neuroendocrine cervical carcinomas classification.

Uterine cervix Neuroendocrine tumors

\section{Low-grade neuroendocrine tumors}

(carcinoid, atypical carcinoid tumor)

High-grade neuroendocrine tumors

(small-cell neuroendocrine carcinoma, large-cell neuroendocrine carcinoma)

Glandular tumor and precursors Adenocarcinoma admixed with neuroendocrine carcinoma

been as definitively established but emerging data suggest a likely association. Alejo et al ${ }^{16}$ explored HPV DNA detection and genotype distribution and their relation to histologic and immunohistochemical features in 49 cervical neuroendocrine tumors. The authors found that $86 \%$ of neuroendocrine tumors had HPV DNA. Single infection (one HPV type) was reported in 98\% of cases. HPV16 was found in $55 \%$ of neuroendocrine tumors, HPV18 in $41 \%$, and $4 \%$ of tumors were positive for other HPV types. HPV18 was four times $(41 \%)$ more frequent in neuroendocrine tumors compared with other histologies $(10 \%)(p<0.001)$. The authors suggested that the greater frequency of HPV18 in both neuroendocrine tumors and adenocarcinomas is indicative of the greater affinity of HPV18 for glandular and neuroendocrine cells compared with other HPV types. They also found that neuroendocrine tumors were more frequently associated with concomitant glandular (rather than squamous) lesions. Neuroendocrine carcinomas showed marked lymphatic permeation, a feature that is particularly characteristic of HPV18-related tumors ${ }^{17}$

Almost all squamous cell carcinomas $(97 \%)$ and half of adenocarcinomas show p16-positive staining. ${ }^{18}$ In the study by Alejo et $a l,{ }^{16}$ the authors found that $p 16$ staining was over-expressed in $86 \%$ of cases. All carcinoid, atypical carcinoid, and large-cell neuroendocrine carcinomas were p16-positive, while $79 \%$ of small-cell carcinomas showed p16-positive staining. Overall, concordant results of p16 and HPV detection were observed in $89 \%$ of cases. In a study by Kuji et al, ${ }^{19}$ the authors evaluated 37 high-grade neuroendocrine carcinomas and found that $72 \%$ had high-risk HPV infections (HPV16 in $14 \%$ and HPV18 in $86 \%$ ). Castle et a ${ }^{20}$ published a metanalysis of 32 studies, including 403 small-cell and nine studies including 45 large-cell neuroendocrine tumors. The authors found that $85 \%$ of small-cell carcinomas were HPV-positive (HPV16 and/or HPV18: 78\%). In a subanalysis of five studies, including 75 patients with small-cell carcinoma, $93 \%$ were positive for $p 16$ by immunohistochemistry and 100\% were HPV-positive. Large-cell neuroendocrine carcinomas were HPV-positive in 88\% (HPV16 and/ or HPV18: $86 \%$ ).

The possible relationship between cervical neuroendocrine carcinomas and HPV infection is a very important finding as these cancers may be prevented by prophylactic HPV vaccines. It also opens the possibility of administering immunotherapy or therapeutic vaccines as a treatment for women with this disease.

\section{IMMUNOHISTOCHEMISTRY}

Immunohistochemistry stains are frequently used to help diagnose neuroendocrine tumors. The most frequently used neuroendocrine stains are chromogranin A, synaptophysin, CD56, and neuron-specific enolase. For large-cell neuroendocrine carcinomas, in addition to morphology by hematoxylin and eosin stain, chromogranin $A$ is the most specific marker to help confirm the diagnosis. Marker positivity in small-cell neuroendocrine tumors ranges from 33\% to $100 \%$. In the latest systematic review published by Tempfer et $a l l^{13}$ the most frequently observed immunohistochemistry markers were synaptophysin $(79 \%)$, neuron specific enolase $(69 \%)$, chromogranin (66\%), and CD56 (61\%). Synaptophysin and CD56 are the most sensitive neuroendocrine markers, but CD56 lacks specificity. Chromogranin is the most specific neuroendocrine marker but lacks sensitivity, with approximately $50 \%$ positivity for small-cell neuroendocrine carcinoma. ${ }^{21}$

In the study by Alejo et $a l,{ }^{16} 65 \%$ were positive for at least one immunohistochemical marker (chromogranin, CD56, and/or synaptophysin) with CD56 being the most frequently positive (62\%) followed by chromogranin (39\%) and synaptophysin (26\%). Similarly, other authors found CD56 to be the most sensitive marker of neuroendocrine differentiation (62\%) followed by chromogranin A (39\%) and synaptophysin (26\%). ${ }^{21}$ Chromogranin positivity may be very focal with punctuate cytoplasmic immunoreactivity, which is only visible on high-power magnification. Small-cell neuroendocrine carcinoma may be only focally positive (often punctuate cytoplasmic staining) or even negative with broad-spectrum cytokeratins. As previously mentioned, most cervical high-grade neuroendocrine carcinomas are diffusely positive for p16 due to the presence of high-risk HPV. ${ }^{21}$

Insulinoma-associated protein 1 (INSM1) may be more specific for neuroendocrine tumors than chromogranin A or synaptophysin. ${ }^{23}$ In a recent publication, 37 high-grade neuroendocrine cases were stained with neuroendocrine markers and INSM1. Chromogranin A and synaptophysin were each expressed in $86 \%$ of cases. INSM1 was detected in $95 \%$ of cases. INSM1 seems to be a useful new neuroendocrine marker and may be associated with the development of high-grade neuroendocrine carcinomas.

Investigators at MD Anderson Cancer Center studied the immunohistochemical expression and prognostic role in survival of HER-2/ neu, epidermal growth factor receptor (EGFR), vascular endothelial growth factor (VEGF), cyclooxygenase-2 (COX-2), estrogen receptor, and progesterone receptor in small- and large-cell neuroendocrine cervical carcinomas in 24 patients. Twenty-three cases (96\%) expressed VEGF, eight (33\%) expressed EGFR, $10(42 \%)$ expressed HER-2/neu, and seven (29\%) expressed COX-2. No significant differences in the expression of these factors were found between small- and large-cell tumors. Only HER-2/neu expression was associated with survival, showing that patients with negative HER-2/neu expression tumors had significantly shorter survival than those whose tumors were positive, 14.2 months versus 33 months, respectively $(p=0.03) .{ }^{24}$

Immunohistochemical expressions may be helpful when trying to determine the site of origin of metastatic small-cell carcinoma. Some studies have shown that $33 \%-84 \%$ of small-cell cervical 


\section{Review Article}

carcinomas exhibit diffuse nuclear positivity for thyroid transcription factor-1 (TTF-1). ${ }^{21}{ }^{25-27}$ Liu et a ${ }^{28}$ compared different expression of neuroendocrine markers, TTF-1, p53, and Ki-67 in 23 cases of cervical small-cell and 56 cases of pulmonary small-cell carcinomas using immunohistochemistry. The TTF-1 expression of small-cell carcinoma of the lung showed significantly higher diffuse and strong positivity in tumor cell nuclei than the cervical counterpart ( $p=0.003)$. Although both tumors had similar morphological features, they have different immunohistochemical panel. Both had similar positivity for CD56 and chromogranin A, but the expression of the synaptophysin in cervical small-cell was significantly higher than in small-cell lung cancer ( $p=0.007)$.

Inmunohistochemistry is important at the time of diagnosis but one should be aware that positive staining could be as low as 33\% using standard markers and that stains may be only focally positive. New markers should be considered if the diagnosis is still unclear as they shown higher positivity rates, as in the case of INMS-1 and VEGF.

\section{MOLECULAR AND IMMUNE PROFILE TESTING}

Although small-cell cervical cancer is a rare tumor without a unifying mutational event, identifying genetic alterations that are amenable to targeted therapy provides an opportunity to individualize therapy especially in relapses where the spectrum of treatments is limited. While high-risk HPV may be involved at an early stage of oncogenesis in many tumors, additional driving events have been postulated to facilitate the progression of small-cell carcinomas. Identification of oncogenic drivers has allowed for a better understanding of the natural history of neuroendocrine tumors. In a recent systematic review, the most common mutations were in p53 (26\%), KRAS (12\%), PIK3CA (18\%), and c-myc (53\%) genes. Loss of heterozygosity was found in $30 \%$ of cases. ${ }^{13}$

Frumovitz et $a{ }^{29}$ identified 44 patients with pure or mixed smallcell cervical cancer. All patients underwent mutational analysis using next-generation sequencing of mutational hotspots in 50 cancer-related genes. Thirty-five mutations were identified in 24 patients (55\%). Most patients (63\%) had one mutation, $29 \%$ had two mutations, and $8 \%$ had three mutations. In all 44 patients, the most common mutations were in PIK3CA $(\mathrm{n}=8 ; 18 \%), K R A S(\mathrm{n}=6$, $14 \%)$, and TP53 $(\mathrm{n}=5,11 \%)$. No other mutation was found in $>7 \%$ of specimens. Of the 24 patients who had a mutation, $21(88 \%)$ had at least one alteration for which there currently exists a class of biological agents targeting that mutation. Although in patients with small-cell cervical cancer no common mutation was found, almost half had at least one actionable mutation for targeted therapy. This is of great importance for patients with recurrent disease where treatment options are limited. Mutational analysis may help to select patients for phase I trials or even off-label therapies.

Our group at MD Anderson Cancer Center recently evaluated the presence of PD-L1 receptors as well as mismatch repair proteins (MLH1, MSH2, MSH6, and PMS2) in specimens of high-grade neuroendocrine cervical cancer. In 23 samples tested for PD-L1 expression, 22 (96\%) were negative for the receptor. In $25 \mathrm{spec}-$ imens evaluated for microsatellite instability, all (100\%) showed intact expression of mismatch repair proteins. These findings suggest a low likelihood of response to PD-1/PD-L1 inhibitors in the recurrent setting. ${ }^{30}$

\section{DIAGNOSIS, STAGING, AND IMAGING}

Cervical neuroendocrine carcinomas are staged using the same International Federation of Gynecology and Obstetrics (FIGO) staging system used for other cervical histologies. The 2018 FIGO staging system allows for imaging and pathologic findings to modify tumor stage.$^{31}$ This is a crucial change for cervical neuroendocrine carcinomas given the higher rate of nodal and distant metastasis at the time of diagnoses. The Society of Gynecologic Oncology (SGO) guideline recommends that given the high rate of distant metastatic disease in neuroendocrine tumors, imaging evaluation should include either a computed tomography (CT) or positron emission tomography (PET)/CT scan. ${ }^{32}$

For disease confined to the cervix both clinically and radiologically, pelvic magnetic resonance imaging (MRI) is considered the best imaging method for tumors greater than $10 \mathrm{~mm}$ to evaluate tumor size and local extension of the disease. ${ }^{33}$ For women with seemingly early-stage disease, we recommend obtaining a pelvic MRI in order to determine if a patient is a candidate for surgical treatment. Another option is transrectal ultrasonography, although this is not commonly utilized at most centers. For other histologic subtypes of cervical cancer (squamous, adenocarcinoma, or adenosquamous), when performed by expert radiologists, the accuracy of transrectal ultrasound compared with MRI in detecting parametrial infiltration was $98.9 \%$ and $94.7 \%(p \leq 0.219)$, respectively. It was considered superior to MRI in detecting small tumors $\left(<1 \mathrm{~cm}^{3}\right)$ (90.5\% vs $81.1 \%$ ( $p \leq 0.049)$, respectively). Transrectal ultrasound was also found to be superior to MRI when evaluating residual tumors after conization $(93.7 \%$ vs $83.2 \%$, respectively; $p \leq 0.006){ }^{34}$

Our approach at MD Anderson Cancer Center is to conduct a thorough gynecologic examination, including a rectovaginal exam, and a PET/CT scan for initial radiologic staging. For patients with disease seemingly limited to the cervix, we recommend either an MRI or ultrasound pre-operatively to evaluate local disease extension. For those patients dispositioned to primary radiation therapy, a pelvic MRI is frequently performed for radiation planning.

We only perform brain imaging in the presence of lung or liver metastases or neurologic symptoms. We strongly encourage physicians to stage patients using the FIGO 2018 system as it might classify patients in a more 'real'/accurate stage according to tumor size, nodal spread, and distant metastases. This allows more tailored treatment and provides patients with a more definitive discussion on prognosis when taking into account nodal status and distant organ involvement (Figure 1).

\section{DISEASE PROGNOSIS}

Several studies have reported age ${ }^{5}$ Iymph node metastases, ${ }^{11}{ }^{35-37}$ race, ${ }^{8}$ smoking, ${ }^{38}$ pure small-cell histology, LVSI, ${ }^{11}$ and tumor size $^{35} 3638$ as independent prognostic factors for neuroendocrine cervical carcinomas. However, stage was the most commonly cited poor prognostic factor in a majority of studies. ${ }^{753-3739}$ Overall, the prognosis of women with neuroendocrine cervical cancer remains poor despite multimodal treatment plans, with a 5-year survival 


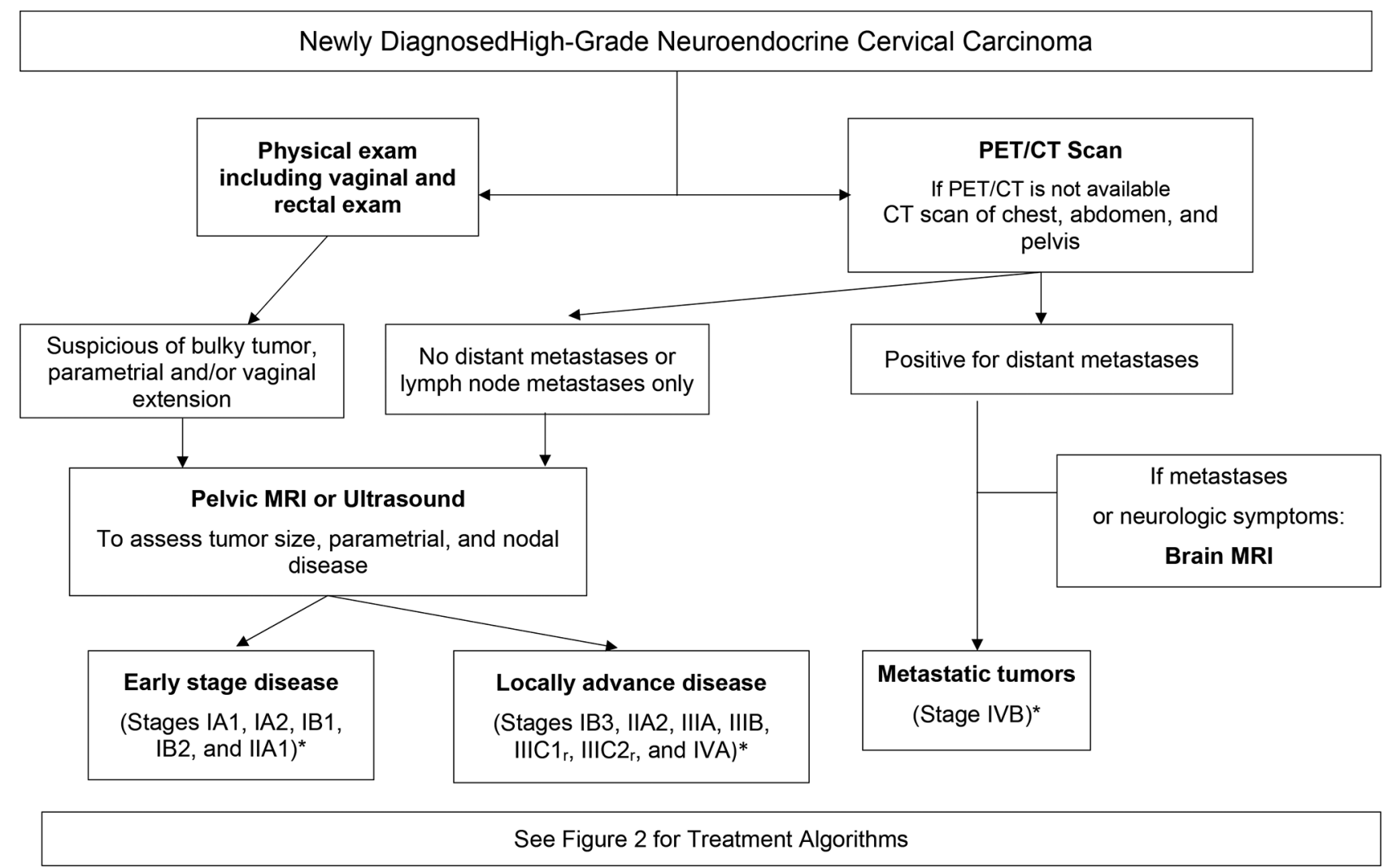

Figure 1 High-grade neuroendocrine cervical carcinoma work-up. MRI, magnetic resonance imaging; PET/CT, positron emission tomography/computed tomography. ${ }^{*} \mathrm{FIGO} 2018$ stage.

rate of $36 \%$ and a median overall survival between 22 and 25 months. ${ }^{5}$ When considering early (I-II) versus advanced stage (IIIIV), the 5 -year overall survival rates are $31 \%-51 \%$ and $0 \%-7 \%$, respectively ${ }^{7840}$ (Table 2).

\section{PRIMARY TREATMENT}

\section{Early-Stage Disease}

In a recently published systematic review that included 3,538 neuroendocrine patients, the most common primary treatment was radical surgery combined with chemotherapy, either as neoadjuvant or adjuvant (40/48 studies). ${ }^{13}$ There was no standard chemotherapy regimen but platinum and etoposide was the most commonly used treatment (24/40 studies). Radiotherapy-based treatment schemes were also commonly utilized in the upfront setting for early-stage disease (15/48 studies).

Both the SGO ${ }^{32}$ and the Gynecologic Cancer Intergroup (GCIG) ${ }^{41}$ recommend multimodal therapy for all stages of neuroendocrine tumors of the cervix, and the majority of patients receive some combination of surgery, radiation, and chemotherapy. For earlystage disease (tumors $\leq 4 \mathrm{~cm}$ ) and negative nodes on imaging, radical hysterectomy and pelvic lymphadenectomy followed by chemotherapy with platinum and etoposide is the primary management recommended with consideration for additional radiotherapy. ${ }^{32}$

\section{Surgery}

There are no prospective studies comparing surgery with chemoradiation therapy for early-stage, resectable tumors. A study from
Ishikawa et $\mathrm{al}^{11}$ of 93 patients with stage I-II high-grade neuroendocrine carcinoma of the cervix showed a median overall survival of 111 months and a disease-free survival of 47 months. There was significant variability as to how patients were treated, but the majority of patients (88; 95\%) underwent radical surgery, and five $(5 \%)$ had definitive radiotherapy. Of the patients receiving surgery, 37 (40\%) underwent radical surgery and pelvic lymphadenectomy with post-operative chemotherapy, $14(15 \%)$ received only surgery, and $25(27 \%)$ received adjuvant radiation or chemoradiation therapy after surgery with or without neoadjuvant chemotherapy before surgery. The hazard ratio (HR) for death for patients who did not undergo surgery as part of their primary treatment was 4.74 (95\% Cl 1.01 to 15.9). Patients who underwent radical surgery had a better overall survival than those who received definitive radiotherapy $(p=0.043)$. The authors found that most clinicians favored radical surgery followed by adjuvant chemotherapy with an etoposide-platinum or irinotecan-platinum regimen as the optimal treatment of choice for stages I-II high-grade neuroendocrine cervical carcinoma. They also concluded that even in early-stage disease, both local recurrence and distant metastasis occurred frequently, and therefore a more effective treatment strategy is required.

Wang et $\mathrm{a}^{\mathrm{f}^{2}}$ reviewed 146 patients with stage I-II disease. Of these, $116(79 \%)$ underwent surgery as part of their primary treatment (primary surgery with/without adjuvant therapy, neoadjuvant chemotherapy plus radical hysterectomy, or peri-operative chemotherapy plus radical hysterectomy). The remaining 30 patients did not undergo surgery but rather had radiation therapy with or without chemotherapy. The authors found there was a trend of worse 
Table 2 Oncologic outcomes of patients with high-grade neuroendocrine cervical carcinoma

\begin{tabular}{|c|c|c|c|c|c|c|c|c|}
\hline Author & Year & $\mathbf{N}$ & Type & Stage & $\begin{array}{l}\text { DFS } \\
\text { (months) }\end{array}$ & OS (months) & $\begin{array}{l}\text { 5-year } \\
\text { DFS (\%) }\end{array}$ & $\begin{array}{l}5 \text {-year } \\
\text { OS (\%) }\end{array}$ \\
\hline Chan $^{38}$ & 2003 & 34 & Multicenter & $\begin{array}{l}\text { I-IIA } \\
\text { IIB-IVB }\end{array}$ & & $\begin{array}{l}31 \\
10\end{array}$ & $\begin{array}{l}32 \\
0\end{array}$ & \\
\hline Weed $^{44}$ & 2003 & 15 & Single institution & All stages & & & 13 & \\
\hline Viswanathan ${ }^{45}$ & 2004 & 21 & Single institution & IB1-IIIB & & 41 & & 29 \\
\hline Chen $^{5}$ & 2008 & 288 & SEER & All stages & & & & 36 \\
\hline \multirow[t]{2}{*}{ Lee $^{52}$} & 2008 & 68 & Multicenter & IB1 & & & & 55 \\
\hline & & & & $\begin{array}{l}\text { IB2-IIA } \\
\text { All stages }\end{array}$ & & 54 & & $\begin{array}{l}32 \\
47\end{array}$ \\
\hline Zivanovic $^{7}$ & 2009 & 17 & Single institution & $\begin{array}{l}\text { All stages } \\
\text { IA1-IB2 } \\
\text { IIB-IV }\end{array}$ & & $\begin{array}{l}21 \\
31 \\
6\end{array}$ & 22 & \\
\hline Cohen $^{10}$ & 2010 & 188 & $\begin{array}{l}\text { SEER + } 52 \text { patients } \\
\text { from four hospitals }\end{array}$ & $\begin{array}{l}\text { I-IIA } \\
\text { IIB-IVA } \\
\text { IVB }\end{array}$ & & & $\begin{array}{l}37 \\
10 \\
0\end{array}$ & \\
\hline Wang $^{42}$ & 2012 & 179 & Multicenter & $\begin{array}{l}\text { All stages } \\
\text { IIB-IVB }\end{array}$ & 16 & 25 (CSS) & $43^{\star} ; 63 \dagger$ & \\
\hline Yin $^{64}$ & 2015 & 23 & Single institution & I-IIIB & & & & 40 \\
\hline Stecklein ${ }^{41}$ & 2016 & 32 & Single institution & All stages & & & 20 & 27 \\
\hline $\operatorname{Lee}^{69}$ & 2016 & 61 & Multicenter & All stages & & 64 & & 36 \\
\hline $\mathrm{Xie}^{65}$ & 2017 & 48 & Single institution & All stages & & 30 & & 31 \\
\hline Ishikawa $11^{11}$ & 2018 & 93 & Multicenter & $\mid A-\| B$ & 47 & 111 & 49 & 55 \\
\hline Tempfer $^{13}$ & 2018 & 3538 & Meta-analysis & All stages & 16 & 40 & & 34 \\
\hline
\end{tabular}

*Primary treatment containing etoposide and platinum for at least five cycles.

†Concurrent chemoradiation with etoposide and platinum for at least five cycles.

CSS, Cancer Surveillance System; DFS, disease-free survival; OS, overall survial; SEER, Surveillance, Epidemiology, and End Results

Program.

failure-free survival ( $41 \%$ vs $61 \%, p=0.086)$ and cancer-specific survival $(48 \%$ vs $62 \%, p=0.122)$ for those women who had radical surgery compared with those who did not undergo surgery.

From our study of patients with stage I-IIA clinically node-negative disease, those who received definitive chemoradiation had significantly better median event-free survival than those who underwent surgery (median not reached vs 18 months, $p=0.04$ ). ${ }^{38}$ Based on the conflicting results from multiple retrospective studies, the role of surgery for early-stage neuroendocrine tumors seems unclear, but certainly surgery alone without adjuvant chemotherapy and/or radiation is not appropriate for any patient with high-grade neuroendocrine carcinomas of the cervix.

\section{Chemotherapy}

Unlike the role of surgery for patients with neuroendocrine tumors, the role of chemotherapy is well established. There is no standard chemotherapy regimen, but due to its pathologic appearances and clinical behaviors similar to small-cell lung cancer, almost all patients with small-cell cervical cancer receive platinum and etoposide as part of their primary therapy. ${ }^{13}$ For early-stage disease, SGO and GCIG guidelines recommend that patients with complete surgical resection undergo adjuvant chemotherapy. ${ }^{32}{ }^{41}$ Zivanovic et $\mathrm{al}^{\boldsymbol{P}}$ reported on 11 patients with early-stage disease (stage IA2-IB2). Of the 11 patients, seven recurred with $86 \%$ of patients having distant recurrence. The 3-year distant recurrence-free survival rate was $83 \%$ for patients who received chemotherapy and $0 \%$ for patients who did not receive chemotherapy as part of their initial treatment $(\mathrm{p}=0.03)$. The estimated 3 -year overall survival rate was $83 \%$ for patients who received chemotherapy and $20 \%$ for patients who did not receive chemotherapy as part of their initial treatment $(p=0.36)$. Ishikawa et $a^{11}$ found that there was an improved disease-free survival in 41 patients who received adjuvant chemotherapy with etoposide-platinum or irinotecan-platinum (HR $0.27,95 \% \mathrm{Cl} 0.10$ to 0.69 ). Also, adjuvant chemotherapy after surgery reduced extra-pelvic recurrences with an OR of $0.37(95 \%$ $\mathrm{Cl} 0.13$ to $0.99, p=0.047)$. A trend toward improved overall survival was also observed when adjuvant chemotherapy was given, but was not statistically significant (HR $0.39,95 \% \mathrm{Cl} 0.15$ to 1.01). Lee et $\mathrm{Af}^{\mathrm{f}}$ reported on a trend in favor of post-operative chemotherapy, and concluded that primary radical surgery followed by adjuvant chemotherapy is the preferred treatment modality for patients with early-stage disease.

Regarding the number of cycles of adjuvant chemotherapy, Pei et a $\left.\right|^{44}$ retrospectively evaluated 92 patients with stage I-II smallcell carcinomas and found that adjuvant chemotherapy with cisplatin and etoposide for at least five cycles was associated with improved 5-year recurrence-free survival compared with other treatments $(68 \%$ vs $21 \%, p<0.001)$. On multivariate analysis, nodal disease $(p<0.003)$, parametrial extension $(p<0.03)$, and cycles of 
etoposide-cisplatin (cisplatin-etoposide $<5$ cycles: $p<0.001$ and no chemotherapy: $p<0.004)$ were independent prognostic factors for disease recurrence.

Pelvic Radiation

A retrospective study including 68 patients with stage IB1-IIA disease reported by Lee et $\mathrm{a}^{\mathrm{A}^{3}}$ suggested that radical hysterectomy followed by adjuvant chemotherapy might be sufficient, as the patients who received chemoradiation did not seem to have a better outcome. In a Japanese multicenter study, the risk of pelvic recurrences after surgery were lower if patients received post-operative radiation (16\%) versus patients who did not undergo radiation (25\%) but the difference did not reach significance (OR 0.61, $95 \% \mathrm{Cl} 0.16$ to 2.01). ${ }^{11}$ Data from MD Anderson Cancer Center showed that the most common sites for first recurrence are outside the pelvis: $38 \%$ in the lungs, $34 \%$ in the liver, and $25 \%$ presenting as carcinomatosis. ${ }^{38}$ Brain recurrence was seen in $25 \%$ of patients, and always in the setting of concurrent lung or liver metastases. If patients received radiation therapy, only $21 \%$ had in-field recurrences.

There seems to be agreement for early-stage disease that chemotherapy is a key component of treatment, but there are conflicting data on the role of combined surgery and chemoradiation.

Protocol at MD Anderson Cancer Center for early-stage disease (tumors $\leq 4 \mathrm{~cm}$ ) and negative nodes on pre-operative imaging is open radical hysterectomy and sentinel lymph node (SLN) biopsies. Post-operatively, our treatment recommendations include adjuvant chemoradiation with concurrent cisplatin and etoposide. Chemotherapy regimen consists of cisplatin $60 \mathrm{mg} / \mathrm{m}^{2}$ on day 1 and etoposide $100 \mathrm{mg} / \mathrm{m}^{2}$ on days 1-3 every 21 days. We decrease the dose of etoposide from 120 to 100 because of the potential toxicity with concurrent radiation. Patients receive two cycles as concurrent chemotherapy, and two to four additional cycles after radiation is completed. Ideally, we aim for patients to be able to receive a total of six cycles. In order to decrease the treatment morbidity associated with post-operative radiotherapy, we only perform SLN mapping and not a full lymphadenectomy when bilateral mapping is achieved (Figure 2).

Prophylactic Cranial Radiation

As treatment for small-cell cervical cancer is derived from the literature on small-cell lung cancer where prophylactic brain irradiation is commonly used for occult metastases, the use of this approach in cervical small-cell tumors has been explored. Weed at al reported in 2003 that $25 \%$ (2/8) of patients with apparent early-stage smallcell cervical cancer developed brain metastases and therefore they proposed prophylactic cranial radiation. ${ }^{45}$ However, neither routine brain CT nor cranial radiation are recommended by the SGO guidelines on initial evaluation of asymptomatic patients for smallcell tumors. In their 14-year retrospective experience Hoskins et al found no cranial metastases. ${ }^{35}$ Data from MD Anderson Cancer Center showed that brain metastases were mostly found $(7 / 8$ patients, $86 \%$ ) when other organ metastases were found. ${ }^{46} 47$

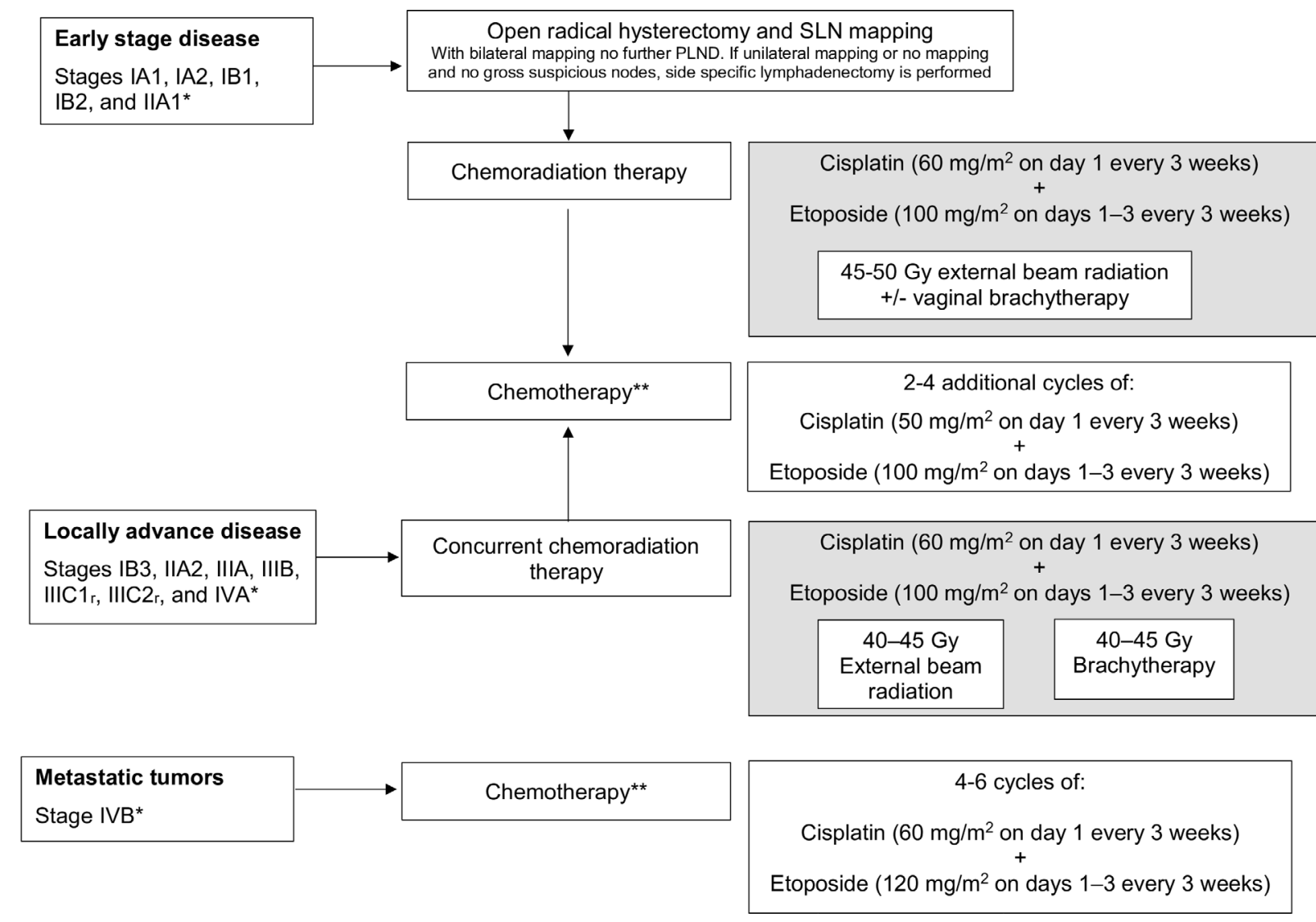

Figure 2 High-grade neuroendocrine cervical carcinoma primary treatment algorithm. *FIGO 2018 stage **If possible six cycles of chemotherapy, with a minimum of four cycles total. PLND, pelvic lymph node dissection; SLN mapping, sentinel lymph node mapping. 


\section{Review Article}

As part of our primary treatment plan, we do not perform prophylactic whole-brain radiation for asymptomatic patients.

\section{Fertility-Sparing Surgery}

With a median age at diagnosis for cervical neuroendocrine tumors of 37 (range 24-77) years, fertility preservation may be a concern both for patients and physicians. ${ }^{46}$ The two primary issues in this patient population are ovarian function (both hormonal and reproductive function) and uterine preservation. As it pertains to the question of ovarian preservation and maintaining ovarian function in patients with locally advanced disease, one may consider ovarian transposition. However, even when doing so, after external beam pelvic radiation and/or brachytherapy, ovarian preservation is only maintained in $65 \%$ of patients. ${ }^{48}$ In addition, particularly in patients with neuroendocrine tumors, there are theoretical concerns regarding residual microscopic disease in the ovary.

There are no data to support the consideration of fertility preservation, such as simple conization or radical trachelectomy, in patients with early-stage disease. Although fertility-preserving surgeries have been reported in women with early-stage smallcell cervical cancer, ${ }^{49-51}$ uterine preservation fertility-sparing treatment is not recommended by the National Comprehensive Cancer Network (NCCN) guidelines for cervical neuroendocrine tumors. ${ }^{52}$ Given that most patients are likely to undergo post-operative radiation therapy it would be unusual to consider fertility-sparing surgery.

Our approach at MD Anderson Cancer Center is to delay starting treatment for 2 to 3 weeks in order to allow for ovarian stimulation and egg retrieval. However, ovarian transposition at the time of radical surgery when the plan is for post-operative radiation or prior to definitive chemoradiation for locally advanced disease seems reasonable if the ovaries appear grossly normal intra-operatively. For young women ( $<50$ years old) who are ultimately made menopausal by surgical and/or radiation therapies, we recommend starting hormone replacement therapy. We strongly recommend against any uterine-preserving surgeries such as simple conization, simple trachelectomy, or radical trachelectomy.

\section{Locally Advanced Disease}

The SGO guideline recommendation for locally advanced disease or non-surgical candidates is a combination of chemotherapy (platinum and etoposide) and radiation. ${ }^{32}$ The most commonly used regimen of cisplatin and etoposide is usually given with cisplatin at 60 to $80 \mathrm{mg} / \mathrm{m}^{2}$ on day 1 and etoposide at 80 to $120 \mathrm{mg} / \mathrm{m}^{2}$ on days 1 to 3 every 21 to 28 days. ${ }^{53-55}$ Carboplatin may be substituted for cisplatin. Other regimens such as paclitaxel and cisplatin, paclitaxel and carboplatin, and the combination of vincristine, cisplatin, and bleomycin have also been reported.

Hoskins et $a^{35}$ first reported that cisplatin and etoposide could be safely administered concurrently with radiation, with additional cisplatin and etoposide given after completing radiation (chemoradiation followed by chemotherapy). A combined modality approach of chemoradiation followed by chemotherapy showed that for stage IB-IVB, concurrent chemoradiation with $\geq 5$ cycles of cisplatin-etoposide was associated with improved 5-year disease-free survival $(63 \%$ vs $13 \%, p=0.025)$ and overall survival $(75 \%$ vs $17 \%$, $\mathrm{p}=0.016) .{ }^{42}$ Distant disease recurrence was the most common type of recurrence $(28 \%)$ and clinical outcome correlated with initial disease extent. The 3-year disease-free survival rate was $55 \%$ and recurrences were unlikely after 3 years. ${ }^{39}$

An abstract presented at ${ }^{55}$ by Bajaj et $a^{56}$ reported on 73 patients with small-cell cervical carcinoma stage IB2-IVA treated with chemoradiation at seven centers in the United States. The median follow-up time was 19 months with $66 \%$ of patients having had a recurrence. Median time to recurrence was 10 months. Recurrence was associated with current smoking (HR 3.32, $p<0.01)$, total equi-effective dose (EQD2) $<50$ Gy vs $71-80$ Gy (HR 3.3, $p=0.07$ ), and no brachytherapy (HR 1.5, $\mathrm{p}=0.25)$. Brain recurrences were seen in $15 \%$ of patients. A decreased hazard of brain recurrence was associated with brachytherapy (HR $0.05, p<0.01$ ), total equi-effective dose (EQD2) >75 Gy (HR 0.11, $p=0.04$ ), and cisplatin and etoposide versus cisplatin alone (HR $0.35, p=0.23$ ). Overall survival for all patients was $48 \%$, favoring concurrent chemoradiation followed by adjuvant chemotherapy versus concurrent chemoradiation only (HR 0.49, $p=0.10$ ). The number of cycles (cisplatin-etoposide) was associated with improved overall survival (HR $0.45, p=0.01$ ) and decreased recurrence (HR 0.67, $\mathrm{p}=0.07$ ). Patients receiving cisplatin-etoposide had improved overall survival ( $59 \%$ vs $44 \%$ ) and a lower recurrence rate $(65 \%$ vs $74 \%$ ) compared with those receiving cisplatin only. Robin et $a^{57}$ identified 100 patients in the National Cancer Data Base (NCDB) with locally advanced non-metastatic neuroendocrine cervical cancer that were treated with definitive chemoradiation between 2004 and 2012. In multivariate analysis, the addition of brachytherapy, compared with external beam radiotherapy alone, was associated with an improved median survival of 49 vs 22 months (HR $0.48,95 \% \mathrm{Cl} 0.25$ to 0.88 ). There was no difference in overall survival for patients treated with neoadjuvant chemotherapy versus patients who received chemotherapy started concurrently with radiation ( $\mathrm{HR} 0.85,95 \% \mathrm{Cl} 0.48$ to 1.50 ).

For women with stage IB3-IVA (FIGO 2018) disease or non-surgical candidates, our recommendation at MD Anderson Cancer Center is chemoradiation with cisplatin and etoposide followed by additional chemotherapy with cisplatin and etoposide for a total of four to six cycles (two cycles with radiation and two to four cycles after radiation completed) with a goal of six total cycles. For women with stage IVB disease, palliative chemotherapy with cisplatin and etoposide is recommended (Figure 2).

\section{RECURRENT DISEASE}

\section{First Recurrence}

For patients with recurrent disease, there is limited consensus on the optimal treatment approach with no standard treatment protocols, and both the SGO and GCIG recommend individualized treatment. ${ }^{32}{ }^{41}$ Often the standard choices include single-agent topotecan, irinotecan, paclitaxel, or docetaxel as these regimens are commonly used to treat recurrent small-cell lung cancer. Our experience at MD Anderson Cancer Center showed that these single-agent regimens had very low activity in patients with recurrent neuroendocrine cervical cancer.

Our regimen of choice for patients with recurrent or progressive disease who have already been treated with a platinum and etoposide combination is a triplet regimen including topotecan, paclitaxel, and bevacizumab given in the same manner as in Gynecologic Oncology Group (GOG) 240, a phase III study 
in patients with recurrent cervical cancer (squamous cell carcinoma, adenocarcinoma, and adenosquamous carcinoma)..$^{58}$ The rationale for this schema is that first this regimen is tolerable in women who have previously undergone definitive chemoradiation. In addition, as single-agent paclitaxel or topotecan are active and frequently used in recurrent small-cell lung cancer, the combination would presumably be equally as active, and potentially more active than the single-agent regimens. Third, small-cell cervical cancers express the vascular epithelial growth factor receptor over $95 \%$ of the time supporting the addition of bevacizumab as an active agent. ${ }^{24}$ Finally, all three drugs have been approved by the US Food and Drug Administration for the treatment of recurrent cervical cancer, thus reimbursement is usually not a concern. Our results with the three-drug combination were published in the largest series of chemotherapy treatments for women with recurrent neuroendocrine cervical carcinoma. ${ }^{59}$ Thirteen patients who received topotecan, paclitaxel, and bevacizumab were compared with 21 patients receiving other regimens, mostly platinum-based regimens with or without taxane. The triplet regimen was associated with a significant improvement in outcome. Median progression-free survival was 8 months versus 4 months for the triplet regimen and other regimens, respectively $(\mathrm{HR} 0.21,95 \% \mathrm{Cl} 0.09$ to 0.54 ). Median overall survival was 9.7 months for the triplet regimen and 9.4 months for patients receiving other regimens (HR $0.53,95 \% \mathrm{Cl} 0.23$ to 1.22 ). For the triplet regimen, $62 \%$ of patients received treatment for $>6$ months and $31 \%$ for $>12$ months versus $19 \%$ and $10 \%$ of those who received other regimens, respectively.

For first recurrence, patients at MD Anderson Cancer Center are typically treated with the three-drug regimen topotecan $(0.75 \mathrm{mg} /$ $\mathrm{m}^{2}$ on days $\left.1-3\right)$, paclitaxel $\left(175 \mathrm{mg} / \mathrm{m}^{2}\right.$ on day 1$)$, and bevacizumab (15 mg/kg on day 1 on a 21-day cycle) (the 'Texas Cocktail'). As part of our strategy for treating patients with recurrent disease, we order molecular testing, $P D-L 1$, and mismatch repair proteins (MLH1, MSH2, MSH6, and PMS2) testing at the time of first recurrence. We use our own institutional platform or other outside providers that offer similar mutational analyses (eg, Foundation Medicine or Caris). As most patients with recurrence will eventually have progressive disease, this testing allows us to triage patients to a targeted therapy either on-protocol or off-label (Figure 3).

\section{Multiple Recurrences}

Immune checkpoint inhibitors and targeted therapies may be beneficial when patients have suffered multiple recurrences; however, the literature is limited in this setting with only three case reports published to date. Paraghamian et al used nivolumab in a patient with recurrent, metastatic, programmed cell death ligand-1 (PD-L1)-negative small-cell neuroendocrine cervical carcinoma, who experienced a complete response ${ }^{60}$ Sharabi et $a^{61}$ reported a patient with metastatic, chemotherapy-refractory neuroendocrine carcinoma with bowel obstruction due to a large tumor burden. Liquid biopsy demonstrated a high number of tumor mutations. The patient was treated with radiotherapy combined with nivolumab and experienced a near-complete systemic resolution of disease for at least 10 months. Our experience with checkpoint inhibitors, however, has been disappointing with no responders (article in preparation). As mentioned above, high-grade neuroendocrine cervical tumors are almost always PD-L1-negative and microsatellite-stable so checkpoint inhibitors should be used with caution. ${ }^{30}$

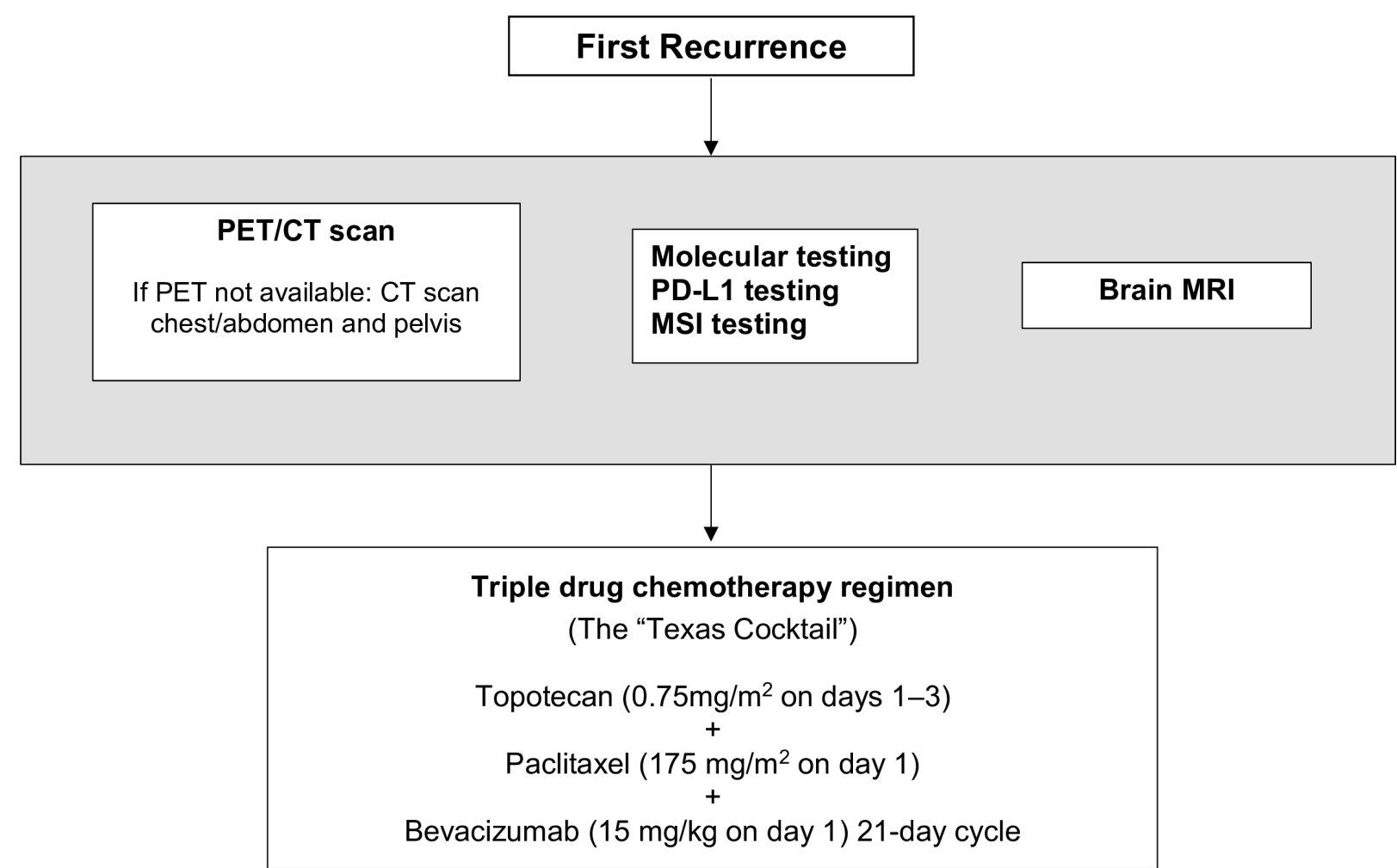

Figure 3 High-grade neuroendocrine cervical carcinoma management algorithm for first recurrence. MRI, magnetic resonance imaging; MSI, mismatch repair proteins (MLH1, MSH2, MSH6, PMS2); PET/CT, positron emission tomography/computed tomography. 


\section{Review Article}

Lyons et $a^{{ }^{2}}$ used the mitogen-activated protein kinase 1 (MEK)-inhibitor trametinib in a woman with recurrent small-cell neuroendocrine cervical carcinoma whose tumor was found to have a $K R A S$ mutation, and had a complete radiologic response after three cycles. ${ }^{62}$

At MD Anderson Cancer Center, for patients whose disease progresses after treatment with topotecan, paclitaxel, and bevacizumab, the recommendation is targeted therapy on a clinical trial. When patients progress after targeted or immunotherapy treatment, we recommend a phase I clinical trial or hospice care.

\section{SURVEILLANCE}

The recommended surveillance for squamous, adenocarcinoma, and adenosquamous carcinomas consists of a physical examination, including a pelvic exam, every 3 to 6 months for 2 years and then every 6 to 12 months for an additional 3 years. In addition, a Papanicolaou test should be performed annually. ${ }^{63}$ For cervical neuroendocrine tumors, the SG0 guidelines recommend physical exam and symptoms review with periodic full-body imaging with either CT or PET/CT scan. ${ }^{32}$ The guideline does not give any recommendation on the frequency of such follow-up and no data exist to make definitive recommendations.

Given the aggressive nature of cervical neuroendocrine tumors, our surveillance plan at MD Anderson Cancer Center consists of a physical examination (with a pelvic exam) every 3 months for the first 2 years and then every 4 to 6 months for the next 3 years. In addition, we order routine radiologic imaging of the chest, abdomen, and pelvis, with either a CT scan or PET/CT scan at every visit.

\section{TUMOR REGISTRY AT MD ANDERSON CANCER CENTER}

Large studies in patients with uncommon or rare tumors are difficult with limited prospective data with which to guide decisions. For women with small- and large-cell neuroendocrine cervical carcinoma there currently exists a rich network on social media sites, making recruitment to protocols much more feasible. ${ }^{64}$ In 2013, we established a Neuroendocrine Cervical Tumor Registry (NeCTuR) at The University of Texas MD Anderson Cancer Center where data are prospectively entered. To date, we have enrolled 252 patients with 110 patients from outside MD Anderson. We continue to enroll and follow patients from around the world. Interested providers may inquire about participation by emailing nectur@mdanderson.org.

Contributors GS: wrote the manuscript, tables, and figures. NG: reviewed the manuscript; provided information on status of NeCTuR database. MF: reviewed, corrected, and medically edited all the manuscript content. AG: reviewed, corrected the manuscript and Figure 1; edited all the chemotherapy information in the manuscript. All authors made substantial contributions to the study conception and design, and/or acquisition of articles for inclusion in the revision. All authors approved the final version of the manuscript prior to submission. Study conception and design: GS and MF. Acquisition of articles to include: NG, GS, MF, and AG. Analysis and interpretation of articles: GS, MF, and AG. Drafting of manuscript: GS, MF, and AG. Critical revision: GS and MF.

Funding This study has been funded by a generous donation from the Allyson Whitney Foundation.

Competing interests None declared.

Patient consent for publication Not required.

Provenance and peer review Commissioned; internally peer reviewed.

\section{REFERENCES}

1. Tavassoli PD FA. Pathology and genetics of tumors of the breast and female genital organs, WHO, IARC Press. Verh Dtsch Ges Pathol 2002;86:116-9.

2. Kurman RJ, Carcangiu ML, Herrington CS YR. WHO classification of tumours of the female reproductive organs. Lyon IARC Press, 2014.

3. Ambros RA, Park JS, Shah KV, et al. Evaluation of histologic, morphometric, and immunohistochemical criteria in the differential diagnosis of small cell carcinomas of the cervix with particular reference to human papillomavirus types 16 and 18. Mod Pathol 1991;4:586-93.

4. Stoler MH, Mills SE, Gersell DJ WA. Small-cell neuroendocrine carcinoma of the cervix. A human papillomavirus type 18-associated cancer. Am J Surg Pathol 1991;15:28-32.

5. Chen J, Macdonald OK, Gaffney DK. Incidence, mortality, and prognostic factors of small cell carcinoma of the cervix. Obstet Gynecol 2008;111:1394-402.

6. McCusker ME, Coté TR, Clegg LX, et al. Endocrine tumors of the uterine cervix: incidence, demographics, and survival with comparison to squamous cell carcinoma. Gynecol Oncol 2003;88:333-9

7. Zivanovic O, Leitao MM, Park KJ, et al. Small cell neuroendocrine carcinoma of the cervix: analysis of outcome, recurrence pattern and the impact of platinum-based combination chemotherapy. Gynecol Oncol 2009;112:590-3.

8. Wang K-L, Yang Y-C, Wang T-Y, et al. Neuroendocrine carcinoma of the uterine cervix: a clinicopathologic retrospective study of 31 cases with prognostic implications. J Chemother 2006;18:209-16.

10. Cohen JG, Kapp DS, Shin JY, et al. Small cell carcinoma of the cervix: treatment and survival outcomes of 188 patients. Am J Obstet Gynecol 2010;203:347.e1-347.e6.

11. Ishikawa M, Kasamatsu T, Tsuda $\mathrm{H}$, et al. Prognostic factors and optimal therapy for stages I-II neuroendocrine carcinomas of the uterine cervix: a multi-center retrospective study. Gynecol Oncol 2018;148:139-46.

12. Gadducci A, Carinelli S, Aletti G. Neuroendrocrine tumors of the uterine cervix: a therapeutic challenge for gynecologic oncologists. Gynecologic Oncology 2017;144:637-46.

13. Tempfer CB, Tischoff I, Dogan A, et al. Neuroendocrine carcinoma of the cervix: a systematic review of the literature. BMC Cancer 2018;18:1-16.

14. Muñoz N, Bosch FX, de Sanjosé S, et al. Epidemiologic classification of human papillomavirus types associated with cervical cancer. $N$ Engl J Med 2003;348:518-27.

15. de Sanjose S, Quint WG, Alemany L, et al. Human papillomavirus genotype attribution in invasive cervical cancer: a retrospective cross-sectional worldwide study. Lancet Oncol 2010;11:1048-56.

16. Alejo M, Alemany L, Clavero $\mathrm{O}$, et al. Contribution of human papillomavirus in neuroendocrine tumors from a series of 10,575 invasive cervical cancer cases. Papillomavirus Res 2018;5:134-42.

17. Lai C-H, Chang C-J, Huang H-J, et al. Role of human papillomavirus genotype in prognosis of early-stage cervical cancer undergoing primary surgery. JCO 2007;25:3628-34.

18. Milde-Langosch K, Riethdorf S, Kraus-Pöppinghaus A, et al. Expression of cyclin-dependent kinase inhibitors p16MTS1, p21WAF1, and p27Kip1 in HPV-positive and HPV-negative cervical adenocarcinomas. Virchows Arch 2001;439:55-61.

19. Kasuga $\mathrm{Y}$, Miyakoshi $\mathrm{K}$, Nishio $\mathrm{H}$, et al. Mid-trimester residual cervical length and the risk of preterm birth in pregnancies after abdominal radical trachelectomy: a retrospective analysis. BJOG: Int J Obstet Gy 2017;124:1729-35.

20. Castle PE, Pierz A, Stoler MH. A systematic review and metaanalysis on the attribution of human papillomavirus (HPV) in neuroendocrine cancers of the cervix. Gynecol Oncol 2018;148:422-9.

21. McCluggage WG, Kennedy K, Busam KJ. An immunohistochemical study of cervical neuroendocrine carcinomas: neoplasms that are commonly TTF1 positive and which may express CK20 and p63. Am J Surg Pathol 2010;34:525-32.

22. Albores-Saavedra J, Latif S, Carrick KS, et al. CD56 reactivity in small cell carcinoma of the uterine cervix. Int J Gynecol Pathol 2005;24:113-7

23. Kuji S, Watanabe R, Sato $Y$, et al. A new marker, insulinomaassociated protein 1 (INSM1), for high-grade neuroendocrine carcinoma of the uterine cervix: analysis of 37 cases. Gynecol Oncol 2017;144:384-90.

24. Tangjitgamol S, Ramirez PT, Sun CC, et al. Expression of HER-2/neu, epidermal growth factor receptor, vascular endothelial growth factor, cyclooxygenase-2, estrogen receptor, and progesterone receptor in small cell and large cell neuroendocrine carcinoma of the uterine 
cervix: a clinicopathologic and prognostic study. Int J Gynecol Cancer 2005;15:646-56.

25. Quinn AM, Blackhall F, Wilson G, et al. Extrapulmonary small cell carcinoma: a clinicopathological study with identification of potential diagnostic mimics. Histopathology 2012;61:454-64.

26. NG O. Value of thyroid transcription factor-immunostaining in distinguishing small cell lung carcinomas from other small cell carcinomas. Am J Surg Pathol 2000;24:1217-23.

27. Carlson JW, Nucci MR, Brodsky J, et al. Biomarker-assisted diagnosis of ovarian, cervical and pulmonary small cell carcinomas: the role of TTF-1, WT-1 and HPV analysis. Histopathology 2007;51:305-12.

28. Liu H, Zhang Y, Chang J, et al. Differential expression of neuroendocrine markers, TTF-1, p53, and Ki-67 in cervical and pulmonary small cell carcinoma. Medicine 2018;97:e11604.

29. Frumovitz M, Burzawa JK, Byers LA, et al. Sequencing of mutational hotspots in cancer-related genes in small cell neuroendocrine cervical cancer. Gynecol Oncol 2016;141:588-91.

30. Carroll M, Salvo G, Ramalingam P, et al. PARP and PD-L1 as potential therapeutic targets for women with neuroendocrine cervical cancer. WAGO 2019.

31. Bhatla N, Aoki D, Sharma DN, et al. Cancer of the cervix uteri. Int J Gynecol Obstet 2018;143(Suppl.3):22-36.

32. Gardner GJ, Reidy-Lagunes D, Gehrig PA. Neuroendocrine tumors of the gynecologic tract: a Society of Gynecologic Oncology (SGO) clinical document. Gynecol Oncol 2011;122:190-8.

33. Hricak H, Gatsonis C, Chi DS, et al. Role of imaging in pretreatment evaluation of early invasive cervical cancer: results of the Intergroup Study American College of Radiology Imaging Network 6651Gynecologic Oncology Group 183. JCO 2005;23:9329-37.

34. Fischerova D, Cibula D, Stenhova $\mathrm{H}$, et al. Transrectal ultrasound and magnetic resonance imaging in staging of early cervical cancer. Int J Gynecol Cancer 2008;18:766-72.

35. Hoskins PJ, Swenerton KD, Pike JA, et al. Small-cell carcinoma of the cervix: fourteen years of experience at a single institution using a combined-modality regimen of involved-field irradiation and platinum-based combination chemotherapy. JCO 2003;21:3495-501.

36. Chang T-C, Lai C-H, Tseng C-J, et al. Prognostic factors in surgically treated small cell cervical carcinoma followed by adjuvant chemotherapy. Cancer 1998;83:712-8.

37. Boruta DM, Schorge JO, Duska LA, et al. Multimodality therapy in early-stage neuroendocrine carcinoma of the uterine cervix. Gynecol Oncol 2001;81:82-7.

38. Chan JK, Loizzi V, Burger RA, et al. Prognostic factors in neuroendocrine small cell cervical carcinoma: a multivariate analysis. Cancer 2003;97:568-74.

39. Hoskins PJ, Wong F, Swenerton KD, et al. Small cell carcinoma of the cervix treated with concurrent radiotherapy, cisplatin, and etoposide. Gynecol Oncol 1995;56:218-25.

40. Sheets EE, Berman ML, Hrountas CK, et al. Surgically treated, earlystage neuroendocrine small-cell cervical carcinoma. Obs Gynecol 1988;71:10-14.

41. Stecklein SR, Jhingran A, Burzawa J, et al. Patterns of recurrence and survival in neuroendocrine cervical cancer. Gynecol Oncol 2016;143:552-7.

42. Wang K-L, Chang T-C, Jung S-M, et al. Primary treatment and prognostic factors of small cell neuroendocrine carcinoma of the uterine cervix: a Taiwanese Gynecologic Oncology Group study. Eur J Cancer 2012;48:1484-94.

43. Pei X, Xiang L, Ye S, et al. Cycles of cisplatin and etoposide affect treatment outcomes in patients with FIGO stage I-II small cell neuroendocrine carcinoma of the cervix. Gynecol Oncol 2017; 147:589-96.

44. Weed JC, Graff AT, Shoup B, et al. Small cell undifferentiated (neuroendocrine) carcinoma of the uterine cervix. J Am Coll Surg 2003;197:44-51.

45. Viswanathan AN, Deavers MT, Jhingran A, et al. Small cell neuroendocrine carcinoma of the cervix: outcome and patterns of recurrence. Gynecol Oncol 2004;93:27-33.

46. Gubbala K, Laios A, Gallos I, et al. Outcomes of ovarian transposition in gynaecological cancers; a systematic review and meta-analysis. J Ovarian Res 2014;7.

47. Lara PN, Natale R, Crowley J, et al. Phase III trial of irinotecan/ cisplatin compared with etoposide/cisplatin in extensive-stage small-cell lung cancer: clinical and pharmacogenomic results from SWOG S0124. JCO 2009;27:2530-5.

48. Singh S, Redline R, Resnick KE. Fertility-sparing management of a stage IB1 small cell neuroendocrine cervical carcinoma with radical abdominal trachelectomy and adjuvant chemotherapy. Gynecol Oncol Rep 2015;13:5-7.

49. Rajkumar S, lyer R, Culora G, et al. Fertility sparing management of large cell neuroendocrine tumour of cervix: a case report \& review of literature. Gynecol Oncol Rep 2016;18:15-17.

50. PY W, Cheng YM, New GH, et al. Case report: term birth after fertility-sparing treatments for stage IB1 small cell neuroendocrine carcinoma of the cervix. BMC Womens Health 2017;17:1-6.

51. NCCN Guidelines Cervical Cancer. Version 32019 - December 17, 20182018.

52. Lee J-M, Lee K-B, Nam J-H, et al. Prognostic factors in FIGO stage IB-IIA small cell neuroendocrine carcinoma of the uterine cervix treated surgically: results of a multi-center retrospective Korean study. Ann Oncol 2008;19:321-6.

53. Hanna N, Bunn PA, Langer C, et al. Randomized phase III trial comparing irinotecan/cisplatin with etoposide/cisplatin in patients with previously untreated extensive-stage disease small-cell lung cancer. JCO 2006;24:2038-43.

54. Noda K, Nishiwaki Y, Kawahara M, et al. Irinotecan plus cisplatin compared with etoposide plus cisplatin for extensive small-cell lung cancer. N Engl J Med 2002;346:85-91.

55. Bajaj A, Frumovitz M, Martin B, et al. Abstracts Selected for 2018 Best of ASTRO (November 30-December 1, 2018) from ASTRO' s 60th Annual Meeting (October 21-24, 2018) Small Cell Carcinoma Cervix Defin. Chemoradiation Local Adv Dis 2018:43-4.

56. Robin TP, Amini A, Schefter TE, et al. Brachytherapy should not be omitted when treating locally advanced neuroendocrine cervical cancer with definitive chemoradiation therapy. Brachytherapy 2016;15:845-50.

57. Tewari KS, Sill MW, Long HJ, et al. Improved survival with bevacizumab in advanced cervical cancer. Obstet Gynecol Surv 2014;69:331-2.

58. Frumovitz M, Munsell MF, Burzawa JK, et al. Combination therapy with topotecan, paclitaxel, and bevacizumab improves progressionfree survival in recurrent small cell neuroendocrine carcinoma of the cervix. Gynecol Oncol 2017;144:46-50.

59. Paraghamian SE, Longoria TC, Eskander RN. Metastatic small cell neuroendocrine carcinoma of the cervix treated with the PD-1 inhibitor, nivolumab: a case report. Gynecol Oncol Res Pract 2017;4.

60. Sharabi A, Kim SS, Kato S, et al. Exceptional response to nivolumab and stereotactic body radiation therapy (SBRT) in neuroendocrine cervical carcinoma with high tumor mutational burden: management considerations from the Center for Personalized Cancer Therapy at UC San Diego Moores Cancer Center. The Oncologist 2017;22:631-7.

61. Lyons YA, Frumovitz M, Soliman PT. Response to MEK inhibitor in small cell neuroendocrine carcinoma of the cervix with a KRAS mutation. Gynecol Oncol Rep 2014;10:28-9.

62. Salani R, Backes FJ, Fung MFK, et al. Posttreatment surveillance and diagnosis of recurrence in women with gynecologic malignancies: Society of Gynecologic Oncologists recommendations. Am J Obstet Gynecol 2011;204:466-78.

63. Zaid T, Burzawa J, Basen-Engquist $\mathrm{K}$, et al. Use of social media to conduct a cross-sectional epidemiologic and quality of life survey of patients with neuroendocrine carcinoma of the cervix: a feasibility study. Gynecologic Oncology 2014;132:149-53.

64. Yin ZM, AJ Y, MJ W, et al. Effects and toxicity of neoadjuvant chemotherapy preoperative followed by adjuvant chemoradiation in small cell neurdendocrine cervical carcinoma. Eur J Gynaecol Oncol 2015;36:326-9

65. Xie S, Song L, Yang F, et al. Enhanced efficacy of adjuvant chemotherapy and radiotherapy in selected cases of surgically resected neuroendocrine carcinoma of the uterine cervix. Medicine 2017:96:e6361.

68. Satoh T, Takei Y, Treilleux I, et al. Gynecologic Cancer Intergroup (GCIG) consensus review for small cell carcinoma of the cervix. Int $J$ Gynecol Cancer 2014;24:S102-S108.

69. Lee DY, Chong C, Lee M, et al. Prognostic factors in neuroendocrine cervical carcinoma. Obstet Gynecol Sci 2016;59. 